\title{
Surfactant-free persistence of surface bubbles in a volatile liquid
}

\author{
Mark Menesses $\odot,{ }^{1}$ Matthieu Roché, ${ }^{2}$ Laurent Royon, ${ }^{2}$ and James C. Bird ${ }^{1, *}$ \\ ${ }^{1}$ Department of Mechanical Engineering, Boston University, Boston, Massachusetts 02144, USA \\ ${ }^{2}$ Matière et Systèmes Complexes, CNRS UMR 7057, Université Paris Diderot, \\ Sorbonne Paris Cité University, F-75013 Paris, France
}

(Received 8 February 2019; published 24 October 2019)

\begin{abstract}
This paper is associated with a video winner of a 2018 APS/DFD Gallery of Fluid Motion Award for work presented at the DFD Gallery of Fluid Motion. The original video is available online at the Gallery of Fluid Motion, https://doi.org/10.1103/APS.DFD.2018. GFM.V0057.
\end{abstract}

DOI: 10.1103/PhysRevFluids.4.100506

Bubbles floating at the free surface of liquids are a common occurrence in everyday life, from washing dishes to enjoying a carbonated beverage. Typically, these bubbles exhibit short-term stability due to the presence of surfactants at the interface. Without these surfactants, existing theory predicts that the length of time over which a bubble will rest at the surface should be governed by a balance of capillary and gravitational drainage resisted by the liquid viscosity $[1,2]$. For example, a bubble at the surface of pure water should (and, indeed, is observed to) pop in a fraction of a second. For these reasons, existing convention holds that to extend the lifetime of a surface bubble, one should either increase the viscosity of the liquid or add surfactants to the system. In this paper we describe a surprising contradiction to this convention where, in spite of a low viscosity and lack of surfactants, bubbles at the surface of pure, highly volatile liquids can persist for several minutes, even hours, without rupture.

The unexpected stability of bubbles at the surface of volatile liquids might be explained as a thermal Marangoni phenomenon. At the surface of a bath of isopropyl alcohol, evaporation readily occurs due to the high volatility of the liquid. The process of evaporation leads to a cooling effect at the interface. When a bubble is present at the surface, evaporation continues at the outer interfaces; however, due to its low thermal mass, the thin film of the bubble can cool much more rapidly than the surrounding bath. This accelerated cooling can give rise to a temperature difference between the bubble cap and liquid bath. The colder bubble cap thus exhibits a higher surface tension which can cause liquid to be drawn from the surrounding bath into the bubble cap by means of a thermal Marangoni flow.

The Marangoni flow into the cap generates a mass flux which is larger than the rate of evaporation. As a result, excess liquid gathers at the apex of the bubble. This pooling of excess liquid is unstable and drains randomly from the top of the bubble to the bath as seen in Fig. 1. This rapid cycle of replenishment and spontaneous drainage leads to small random motions of the bubble when viewed with the naked eye.

*Corresponding author: jbird@bu.edu

Published by the American Physical Society under the terms of the Creative Commons Attribution 4.0 International license. Further distribution of this work must maintain attribution to the author(s) and the published article's title, journal citation, and DOI. 


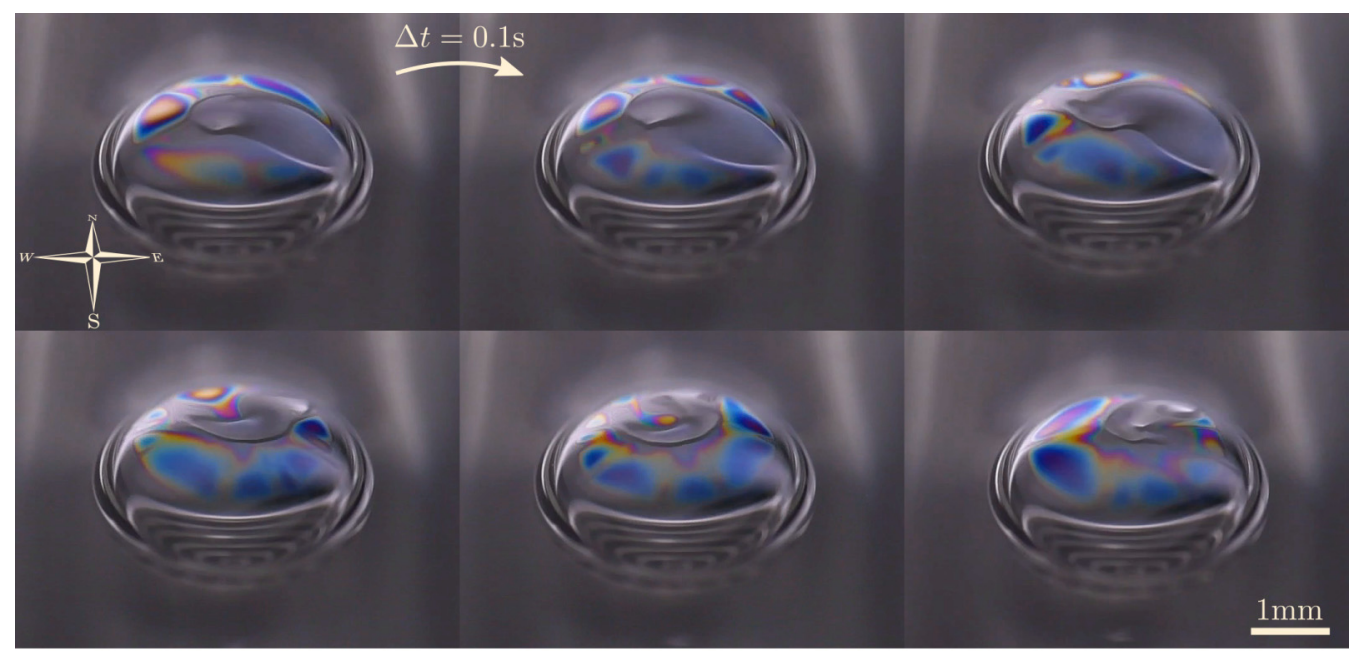

FIG. 1. An air bubble at the surface of isopropyl alcohol that persists for several minutes. A dazzling array of colors displayed over the bubble cap results from white light interference. These interference fringes indicate that the liquid film is on the order of 100-1000 nm in thickness over the majority of the bubble cap. A notable feature is seen on the east face of the bubble in the first frame, where the interference fringes disappear, hinting that the region is much thicker. It is in this thicker region where excess liquid that has been advected into the cap subsequently drains back into the liquid bath. The third and fourth frames highlight the rapid and stochastic change of direction of this drainage flow, where it has switched from the east face to the north face of the bubble. The time between frames is $0.1 \mathrm{~s}$.

Since temperature differences are necessary to drive this phenomenon, we view the system with standard and infrared imaging simultaneously, as seen in Fig. 2. Figure 2(a) shows a side view of the bubble cap, where excess liquid can be seen at the apex and draining down in a small portion of the cap. In the simultaneously captured thermographic image in Fig. 2(b), a difference in temperature

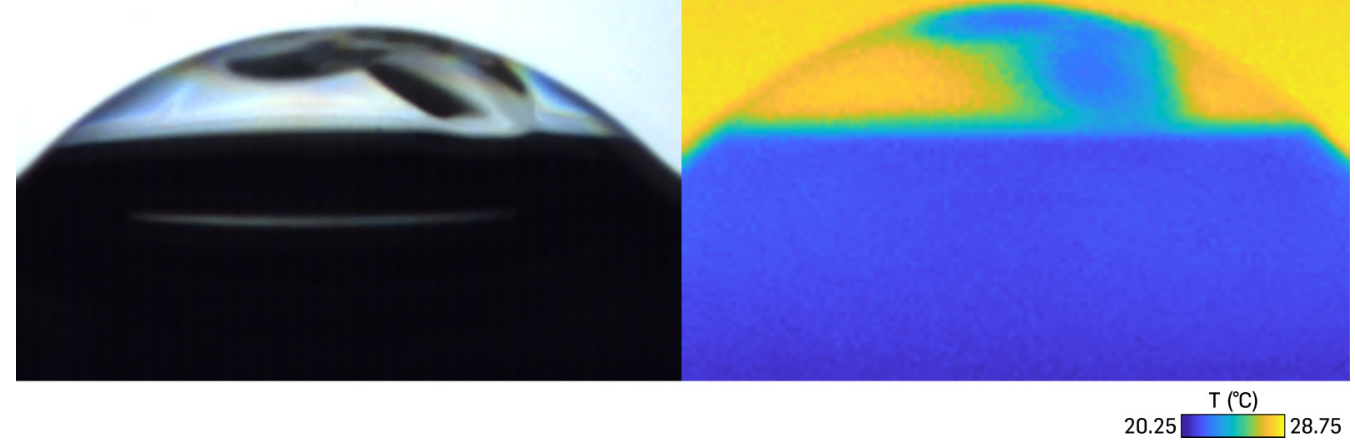

FIG. 2. A side view of a persisting air bubble at the surface of a bath of isopropyl alcohol. (a) Using standard imaging, we see the pooling of excess liquid at the apex of the bubble cap and drainage of this liquid down the front of the bubble. (b) Infrared imaging showing a temperature difference within the drainage region. 
can be seen in this region of pooled, cooled liquid. Although the Marangoni effect appears to rationalize the existence of these surfactant-free yet persistent bubbles in volatile liquids, further study is necessary to elucidate the limit to which these thermal Marangoni stresses might stabilize free surface bubbles in pure liquids of varying volatility.

M.M. acknowledges support from the National Science Foundation Graduate Research Fellowship Program under Grant No. DGE-1247312 and an international travel allowance through the Graduate Research Opportunities Worldwide (GROW). J.C.B. acknowledges partial support from the National Science Foundation under Grant No. 1351466. Any opinions, findings, and conclusions or recommendations expressed in this material are those of the authors and do not necessarily reflect the views of the National Science Foundation. We acknowledge early contributions to this work by D. Holmes and H. A. Stone in 2009.

[1] P. Howell, The draining of a two-dimensional bubble, J. Eng. Math. 35, 251 (1999).

[2] G. Debrégeas, P.-G. De Gennes, and F. Brochard-Wyart, The life and death of 'bare' viscous bubbles, Science 279, 1704 (1998). 